\title{
MODULES WITH THE QUASI-SUMMAND INTERSECTION PROPERTY
}

\author{
Ulrich Albrecht and Jutta Hausen
}

\begin{abstract}
Given a torsion-free abelian group $G$, a subgroup $A$ of $G$ is said to be a quasisummand of $G$ if $n G \leqslant A \oplus B \leqslant G$ for some subgroup $B$ of $G$ and some positive integer $n$. If the intersection of any two quasi-summands of $G$ is a quasi-summand, then $G$ is said to have the quasi-summand intersection property. This is a generalisation of the summand intersection property of $\mathrm{L}$. Fuchs. In this note, we give a complete characterisation of the torsion-free abelian groups (in fact, torsion-free modules over torsion-free rings) with the quasi-summand intersection property. It is shown that such a characterisation cannot be given via endomorphism rings alone but must involve the way in which the endomorphism ring acts on the underlying group. For torsion-free groups $G$ of finite rank without proper fully invariant quasi-summands however, the structure of its quasi-endomorphism ring $\mathbf{Q} E(G)$ suffices: $G$ has the quasi-summand intersection property if and only if the ring $\mathrm{Q} E(G)$ is simple or else $G$ is strongly indecomposable.
\end{abstract}

\section{INTRODUCTION}

Irving Kaplansky observed that for a free module over a principal ideal domain, the intersection of any two direct summands is again a direct summand [18]. Problem 9 of Laszlo Fuchs' monograph Infinite Abelian Groups asked for a characterisation of the abelian groups $G$ which have the summand intersection property (abbreviated by SIP in $[\mathbf{2 2}])$, that is, have the property that the intersection of any two direct summands is a direct summand. For modules over arbitrary rings, this problem was considered by George V. Wilson in [22], and a solution was given by Arnold and Hausen in [5]. As is to be expected, the summand intersection property is not preserved under quasi-isomorphisms. That led to some unsatisfactory results: if $G$ is a finite rank torsion-free abelian group with SIP that has no proper fully invariant direct summands, then $G$ must be a direct sum of pairwise quasi-isomorphic groups whose common quasi-endomorphism ring is a division algebra [15]. The converse of this, however, is false. This was one reason to consider the quasi-summand intersection property (q-SIP for short) instead: an $R$-module $M$ has q-SIP if the intersection of any two quasi-summands is a quasi-summand. We

Received 5th September, 1990.

Copyright Clearance Centre, Inc. Serial-fee code: 0004-9729/91 \$A2.00+0.00. 
will show that a torsion-free abelian group $G$ has q-SIP if and only if (i) its endomorphism ring $E=E(G)$ has q-SIP as a right module over itself, and (ii) $G$ satisfies a mild flatness condition as a left $E$-module (2.7). A torsion-free ring $R$ has q-SIP as a right regular module if and only if the ring $Q \otimes_{z} R$ has SIP as a right regular module (2.3); in particular, if $G$ is flat as left $E$-module, the conditions of $G$ having q-SIP, its endomorphism ring having q-SIP, and its quasi-endomorphism ring having SIP are all equivalent (2.8). While every completely decomposable torsion-free abelian group with q-SIP must be flat over its endomorphism ring (3.4), this need not be the case for torsion-free groups with q-SIP in general (4.3). A finite-rank torsion-free abelian group $G$ which is quasi-decomposable but without fully invariant quasi-summands has q-SIP if and only if its quasi-endomorphism ring is a simple ring (3.2). It follows that modules with q-SIP need not have SIP [15, p.144]. We will show that SIP does not imply q-SIP either (4.4) so that both properties are independent of each other. Finally, as was the case with SIP, it is not possible to characterise the q-SIP via endomorphism rings alone: using a representation theorem due to Faticoni and Goeters [13] we will show the existence of two torsion-free abelian groups $G$ and $H$ of finite rank with $E(G) \simeq E(H)$ such that $G$ has q-SIP while $H$ does not (4.5). Thus, in addition to the structure of its endomorphism ring, the way in which the endomorphism ring acts on the group must enter into the characterisation.

\section{Modules With Q-SIP}

As usual, $Z$ and $Q$ denote the ring of integers and field of rational numbers, respectively.

The word ring is used to mean ring with identity. Throughout, $R$ will be a torsionfree ring (that is, the additive group $R^{+}$of $R$ is torsion-free) and $M=M_{R}$ is a unital right $R$-module such that the group $(M,+)$ is torsion-free. Let $Q R=Q \otimes z R$ and $Q M=Q \otimes z M$. Then $Q R$ is an associative $Q$-algebra [7], $Q M$ is a right module over $R$ as well as a right $Q R$-module [9, p.23], and both $R$ and $M$ are naturally embedded in $Q R$ and $Q M$, respectively [9, p.130, 2.3]. We will simplify notation by writing $q a$ for $q \otimes a$. If $K$ is an $R$-submodule of $Q M$ then $Q K$ is the $Q$-subspace of $Q M$ generated by $K$, that is, the set of all rational multiples of elements in $K$.

Let $\mathcal{L}$ be the ring of all $Q R$-endomorphisms of $Q M$. The ring $E=E_{R}(M)$ of all $R$-endomorphisms of $M$ may be regarded as the subring $\mathcal{L}$ consisting of all $\varepsilon \in \mathcal{L}$ such that $\varepsilon M \leqslant M$. If $K \leqslant M$ then $\operatorname{Hom}_{R}(M, K)$ is a right ideal of $E$. We let $Q \operatorname{Hom}_{R}(M, K)$ be the $Q$-subspace of $\mathcal{L}$ generated by $\operatorname{Hom}_{R}(M, K)$. In particular, the quasi-endomorphism ring $Q E=Q E_{R}(M)$ consists of all rational multiples of endomorphisms of $M$.

Given two $R$-submodules $K$ and $L$ of $Q M$, the terms "quasi-contained" and 
"quasi-equal" are used in the abelian group sense [14, p.148]: $K \leqslant L$ if $n K \subseteq L$ for some natural number $n$, and $K \doteq L$ if both $K \leqslant L$ and $L \leqslant K$. Clearly, if $K \doteq L$ then $Q K=Q L$. Two torsion-free abelian groups $G$ and $H$ are quasi-isomorphic, in symbol $G \dot{\check{ }} H$, if they are isomorphic to quasi-equal groups. Furthermore, an $R$-submodule $K$ of $Q M$ is a quasi-summand of $M$ if $K \doteq \pi(M)$ for some idempotent $\pi$ in $Q E_{R}(M)$. If $A$ is an $R$-submodule of $Q M$ then $A$ is a quasi-summand of $M$ if and only if $M \doteq A \oplus B$ for some $R$-submodule $B$ of $M$. Also, if $M \doteq K \oplus L$ and $K \leqslant A \leqslant M$ then $A \doteq K \oplus(A \cap L)$, and the relation of being a quasi-summand is transitive.

We summarise a few technical results:

LEMMA 2.1. Let $S$ be a torsion-free ring and let $\pi_{1}, \pi_{2}$ and $\pi$ be idempotents in $Q S$. Then

(1) $S \cap \pi Q S \doteq \pi S$.

(2) $\pi_{1} S \cap \pi_{2} S \doteq \pi S \Longleftrightarrow \pi_{1} Q S \cap \pi_{2} Q S=\pi Q S$.

(3) If $S=E_{R}(M)$ and $\pi_{1} M \cap \pi_{2} M \doteq \pi M$ then $\pi_{1} S \cap \pi_{2} S \doteq \pi S$.

Proof: Easy calculations establish (1) and (2). For (3), note that the hypothesis implies $\pi Q M=Q\left(\pi_{1} M \cap \pi_{2} M\right)=\pi_{1} Q M \cap \pi_{2} Q M$. Consequently, $\pi=\pi_{i} \pi$ and $\pi Q S \subseteq \pi_{1} Q S \cap \pi_{2} Q S$. If $\sigma \in \pi_{1} Q S \cap \pi_{2} Q S$ then $\sigma Q M \subseteq \pi Q M$ so that $\sigma=\pi \sigma \in \pi Q S$. Hence $\pi Q S=\pi_{1} Q S \cap \pi_{2} Q S$. Since $S$ is a torsion-free ring, (2) is applicable.

The following result is essential. It will be used throughout the paper without necessarily being mentioned. It is the counterpart to the fact that an $R$-module $M$ has SIP if and only if, given any decomposition $M=A \oplus B$ and any $\varepsilon \in \operatorname{Hom}_{R}(A, B)$, the kernel of $\varepsilon$ is a direct summand $[22,15]$.

Proposition 2.2. The $R$-module $M$ has q-SIP if and only if, given any quasi-decomposition $M \doteq A \oplus B$ and any $R$-homomorphism $\varepsilon: A \rightarrow B$, the kernel of $\varepsilon$ is a quasi-summand of $A$.

Proof: Assume, firstly, that $M \doteq A \oplus B$ has q-SIP and let $\varepsilon: A \rightarrow B$. Consider $T=\{a+\varepsilon(a) \mid a \in A\}$. Then $A \oplus B=T \oplus B$. By hypothesis, $A \cap T=\operatorname{kernel}(\varepsilon)$ is a quasi-summand of $M$ and, thus, of $A$. Conversely, assume $M$ satisfies the stated condition and let $\pi_{i} \in Q E_{R}(M)$ be idempotents, $i=1,2$. Choose a positive integer $n$ such that $n \pi_{i} \in E_{R}(M), i=1,2$. Then $n \pi_{i} M \oplus n\left(1-\pi_{i}\right) M \leqslant M \leqslant \pi_{i} M \oplus\left(1-\pi_{i}\right) M$, $i=1,2$. Let $\varepsilon=n^{2}\left(1-\pi_{1}\right) \pi_{2} \mid \pi_{1} M$. Then $\varepsilon: \pi_{1} M \rightarrow\left(1-\pi_{1}\right) M$ and one verifies that $\operatorname{kernel}(\varepsilon) \doteq\left(\pi_{1} M \cap \pi_{2} M\right) \oplus\left(\pi_{1} M \cap\left(1-\pi_{2}\right) M\right)$. Since, by hypothesis, the kernel of $\varepsilon$ is a quasi-summand of $\pi_{1} M$, so is $\pi_{1} M \cap \pi_{2} M$.

It follows from 2.2 that $q$-SIP is inherited by quasi-summands.

As in [5], we investigate the quasi-summand intersection property in the case that $M=R$ : 
Proposition 2.3. The following properties of the torsion-free ring $R$ are equivalent.

(1) $R$ has $q$-SIP as a right $R$-module.

(2) $Q R$ has SIP as a right $Q R$-module.

(3) For all idempotent $\pi \in Q R$ and all $x \in(1-\pi) Q R \pi$, the right ideal $x Q R$ is projective.

Proof: The equivalence of (2) and (3) is contained in Proposition 3 of [5]; the equivalence of (1) and (2) follows from 2.1(2) above.

As in [5], this leads to the discussion of right principal projective rings: a ring $R$ is said to be right principal projective (or right p.p. [10]) if every principal right ideal of $R$ is projective as a right $R$-module. We obtain at once

COROLlary 2.4. Let $R$ be a torsion-free ring. If $Q R$ is right principal projective then $R_{R}$ has $q-S I P$.

In order to apply 2.3 to endomorphism rings, it is desirable to characterise the principal projective right ideals of quasi-endomorphism rings. The $M$-socle of an $R$ module $K$ is defined to be $S_{M}(K)=\sum\left\{\sigma M \mid \sigma \in \operatorname{Hom}_{R}(M, K)\right\}$.

Proposition 2.5. Let $E=E_{R}(M)$, let $\varepsilon \in E$ and let $K=\operatorname{kernel}(\varepsilon)$. The following conditions are equivalent.

(1) The right ideal $\varepsilon Q E$ is projective.

(2) There exists an idempotent $\pi \in Q E$ such that $\operatorname{Hom}_{R}(M, K) \doteq \pi E$.

(3) The $M$-socle $S_{M}(K)$ of $K$ is a quasi-summand of $M$.

PROOF: Let $\varepsilon^{*}: Q E \rightarrow \varepsilon Q E$ be the left multiplication by $\varepsilon$. One verifies that $\operatorname{kernel}\left(\varepsilon^{*}\right)=Q \operatorname{Hom}_{R}(M, K)$, and $\varepsilon Q E$ is projective if and only if $Q \operatorname{Hom}_{R}(M, K)=$ $\pi Q E$ for some idempotent $\pi \in Q E$. Thus, (2) implies (1). Assume (1). Then we have $Q \operatorname{Hom}_{R}(M, K)=\pi Q E$ which implies that, for each $\sigma \in \operatorname{Hom}_{R}(M, K), \sigma=\pi \sigma$. Hence $S_{M}(K) \leqslant \pi M$. If $m$ is a nonzero integer with $m \pi \in \operatorname{Hom}_{R}(M, K)$ then $m \pi M \leqslant$ $S_{M}(K)$. Thus, (1) implies (3). Assume (3). Then $S_{M}(K) \doteq \pi M$ with $\pi=\pi^{2} \in Q E$. There exists a positive integer $n$ such that $n \pi M \leqslant S_{M}(K) \leqslant K$ and $n S_{M}(K) \leqslant \pi M$. Hence $n \pi \in \operatorname{Hom}_{R}(M, K)$ so that $n \pi E \leqslant \operatorname{Hom}_{R}(M, K)$; for every $\sigma \in \operatorname{Hom}_{R}(M, K)$, $n \sigma M \leqslant n S_{M}(K) \leqslant \pi M$ which implies $\sigma=\pi \sigma \in \pi E$. Thus $\operatorname{Hom}_{R}(M, K) \doteq \pi E$ as desired.

COROLlary 2.6. The kernel $K$ of an endomorphism $\varepsilon \in E_{R}(M)=E$ is a quasi-summand of $M$ if and only if the right ideal $\varepsilon Q E$ is projective and $K \doteq S_{M}(K)$.

We now give a characterisation of the $R$-modules $M$ with q-SIP in terms of their quasi-endomorphism ring. If $I$ is a right ideal of $E=E_{R}(M)$ we let $I M=$ $\sum\{\varphi M \mid \varphi \in I\}$. Clearly, if $J$ is a right ideal of $E$ with $I \doteq J$ then $I M \doteq J M$. 
Note that, if $K \leqslant M$, then $\operatorname{Hom}_{R}(M, K)$ is a right ideal of $E_{R}(M)$ and the $M$-socle $S_{M}(K)=\operatorname{Hom}_{R}(M, K) M$.

THEOREM 2.7. The following properties are equivalent for a right $R$-module $M$ with endomorphism ring $E$ :

(1) $M$ has q-SIP.

(i) $E$ has q-SIP as a right $R$-module;

(ii) for all idempotents $\pi$ and $\rho$ in $Q E, \pi M \cap \rho M \doteq(\pi E \cap \rho E) M$.

(i) $Q E$ has $S I P$ as a right $Q E$-module;

(ii) if $M \doteq A \oplus B$ and $\varphi \in H_{o m_{R}}(A, B)$ then $\operatorname{kernel}(\varphi)$ is quasiequal to its $A$-socle.

(4) If $\pi \in Q E$ is idempotent and $\epsilon \in(1-\pi) E \pi$ then

(i) the right ideal $\varepsilon Q E$ is projective; and

(ii) the kernel of $\varepsilon \mid M$ is equal to its $M$-socle.

Proof: The quasi-summands of the right $E$-module $E$ are precisely the $E$ submodules $J$ of $Q E$ of the form $J \doteq \pi E$ for some idempotent $\pi \in Q E$. It follows from 2.1(3) that (1) implies (2). For the reverse implication, let $\pi_{i}$ be two idempotents in $Q E$. Then there is $\pi=\pi^{2} \in Q E$ with $\pi_{1} E \cap \pi_{2} E \doteq \pi E$, and $\pi M=(\pi E) M \doteq\left(\pi_{1} E \cap \pi_{2} E\right) M \doteq \pi_{1} M \cap \pi_{2} M$. Thus (1) and (2) are equivalent; because of 2.2 and 2.3 they imply (3). Note that $\varepsilon \in(1-\pi) Q E \pi$ with $\pi=\pi^{2} \in Q E$ is equivalent to $M \doteq A \oplus B$ for some $A$ and $B$ with $\varepsilon(A) \leqslant B$ and $\varepsilon(B)=0$. Using 2.2 and 2.5, this implies the equivalence of (1) and (4). Finally, assume (3). Then there exists an idempotent $\pi \in Q E$ and an $\varepsilon \in(1-\pi) Q E \pi$ such that $\varepsilon \mid A=\varphi$. By 2.3 , the right ideal $\varepsilon Q E$ is projective so that the $M$-socle of its kernel is a quasi-summand, by 2.5 ; hence $\operatorname{kernel}(\varphi)$ is a quasi-summand of $A$ completing the proof.

We now turn to the conditions (ii) in Theorem 2.7. These are weakened flatness conditions considered by various authors: Arnold showed that an abelian group $A$ is flat as a module over its endomorphism ring $E$ if and only if (a) for every $\epsilon \in E$ the kernel of $\varepsilon$ equals its $A$-socle, and (b) $(I \cap J) A=I A \cap J A$ for all finitely generated right ideals $I$ and $J$ of $E$ [4, Theorem 1.1]. The analogue of this for $R$-modules is contained in [5, Proposition 10]. Ulmer proved that an $R$-module $M$ is flat over its endomorphism ring if and only if, given any positive integer $n$ and any homomorphism $\varphi: M^{n} \rightarrow M$, the kernel of $\varphi$ equals its $M$-socle [21, p.531]. Thus, if $M$ is flat over $E_{R}(M)$, the conditions (ii) in 2.7 are automatically satisfied:

Corollary 2.8. Suppose that the $R$-module $M$ is flat as module over its endomorphism ring $E=E_{R}(M)$. Then the following conditions are equivalent.

(1) $M$ has $q$-SIP.

(2) E has q-SIP as a right $E$-module. 
(3) $Q E$ has SIP as a right $Q E$-module.

(4) For every idempotent $\pi \in Q E$ and every $\varepsilon \in(1-\pi) E \pi$, the right ideal $\varepsilon Q E$ is projective.

The following result indicates a source for modules with both intersection properties:

Corollary 2.9. Suppose the $R$-module $M$ is flat over its endomorphism ring $E_{R}(M)$ and $E_{R}(M)$ is right principal projective. Then $M$ has both SIP and q-SIP.

ProOF: $E$-flat modules with right principal projective endomorphism rings have SIP [5, Corollary 12]. Since $Q E_{R}(M)$ is right principal projective if $E_{R}(M)$ is, the assertion follows from 2.4 and 2.8 .

If $M$ is a module such that $M^{n}$ has q-SIP for every positive integer $n$, it does not follow that each $M^{n}$ has SIP; this can be seen from the $Z$-module $M=Z \oplus A$ where $A$ is any noncyclic subgroup of $Q$ (see 3.6 below and $[15,5.1]$ ). The following result indicates that modules with q-SIP are more common than modules with SIP:

Proposition 2.10. If $M$ is an $R$-module such that $M^{n}$ has $S I P$ for every natural number $n$ then $M^{n}$ has $q$-SIP for every natural number $n$.

Proof: Put $E=E_{R}(M)$ and assume that the $M^{n}$ has SIP for all positive integers $n$. Then, for each positive integer $m$ and every homomorphism $\varphi: M^{m} \rightarrow M$, the kernel of $\varphi$ is a direct summand and, thus, equals its $M$-socle. By Ulmer's theorem [21, p. 531] this implies $M$ is $E$-flat. Proceed as in [1, p.567, proof of 2.2] to see that $E$ is right semihereditary. (Indeed, Theorem 2.2 of [15] is valid without the hypothesis that the module $A$ be indecomposable.) Let $k$ be a positive integer. Since the module $M^{k}$ satisfies the hypothesis, too, it follows that $M^{k}$ is a flat $E_{R}\left(M^{k}\right)$-module and $E_{R}\left(M^{k}\right)$ is right semihereditary, hence right p.p. Apply 2.9.

\section{TORSION-FREE ABELIAN GROUPS WITH Q-SIP}

All groups under consideration will be torsion-free and abelian. It was Bjarni Jónsson who introduced the concept of quasi-isomorphism and showed that in the category of finite-rank torsion-free abelian groups with the quasi-homomorphisms as morphisms a Krull-Schmidt theorem is valid [17] (see [14, 92.5]). A group $A$ is strongly indecomposable if every nonzero quasi-summand of $A$ is quasi-equal to $A$. Obviously, every strongly indecomposable group has q-SIP.

Let $G$ be a torsion-free abelian group of finite rank. Then $G \doteq A_{1} \oplus \cdots \oplus A_{n}$ where each of the $A_{i}$ is strongly indecomposable. If each $A_{i}$ is fully invariant in $G$ (that is, $\varepsilon\left(A_{i}\right) \leqslant A_{i}$ for every $\varepsilon \in E(G)$ ) then $G$ has q-SIP (see [3, 2.1]). We shall consider the case that $G$ is without proper fully invariant quasi-summands but not 
strongly indecomposable. Such a group will be shown to have q-SIP if and only if its endomorphism ring is simple. For the proof of this we need the following result which is essentially contained in [6]. Note that we do not require the rank to be finite.

PROPOSITION 3.1. Let $W$ be a torsion-free abelian group such that $Q E(W)$ is a semisimple ring. Then the following hold:

(1) If $I$ is a right ideal of $E(W)$ such that $Q I W=Q W$ then $n \cdot 1_{W} \in I$ for some natural number $n$.

(2) For every subgroup $L$ of $W, S_{W}(L)$ is a quasi-summand of $W$.

(3) If $H$ is a torsion-free group with $S_{W}(H) \doteq H$ and $\varepsilon: H \rightarrow W$ is an epimorphism then the kernel of $\varepsilon$ is a quasi-summand of $H$.

Proof: Assume the hypothesis of (1). Then $Q I$ is a right ideal of $Q E=Q E(W)$ and the latter is semisimple so that $Q I=\pi Q E$ for some idempotent $\pi \in Q E$. Hence $Q W=\pi Q W$ which implies $\pi=1$ and $1 \in Q I$ as desired. For (2), assume $L \leqslant W$ and let $I=\operatorname{Hom}(W, L)$. Then $I$ is a right ideal of $E(W)$ and $S_{W}(L)=I W$. As before, the semisimplicity of $Q E$ implies the existence of $\pi=\pi^{2} \in Q E$ with $Q I=\pi Q E$. It follows that $\sigma=\pi \sigma \in \pi E$ for all $\sigma \in I$. If $n$ is a nonzero integer with $n \pi \in I$ then $n \pi E \leqslant I \leqslant \pi E$ and hence $n \pi W \leqslant I W=S_{W}(L) \leqslant \pi W$. In order to prove (3), let $I=\varepsilon \operatorname{Hom}(W, H)$. Then $I$ is a right ideal of $E$ and $H \doteq S_{W}(H)=\operatorname{Hom}(W, H) W$ which implies $W=\varepsilon H \doteq I W$. Part (1) completes the proof.

THEOREM 3.2. Let $G$ be a torsion-free abelian group of finite rank which is not strongly indecomposable. The following conditions are equivalent.

(1) $G$ has q-SIP and contains no proper fully invariant quasi-summands.

(2) $G \dot{\sim} A^{n}$ for some positive integer $n$ and some group $A$ such that $Q E(A)$ is a division algebra.

(3) The quasi-endomorphism ring $Q E(G)$ is a simple (Artinian) ring.

(4) For every natural number $m$, the group $G^{m}$ has $q$-SIP and contains no. proper fully invariant quasi-summands.

Proof: It suffices to show that the first three conditions are equivalent. In order to show that (1) implies (2), assume, by way of contradiction, that $G$ is quasidecomposable but not of the stated form. Since the quasi-summand intersection property is inherited by quasi-summands, we may assume that $G \doteq A_{1} \oplus \cdots \oplus A_{n}$ with each $A_{i}$ strongly indecomposable, $A_{i}$ and $A_{j}$ not quasi-isomorphic for $i \neq j$ and $n \geqslant 2$. By hypothesis, for each $i$ there exists a $j \neq i$ such that $\operatorname{Hom}\left(A_{i}, A_{j}\right) \neq 0$ so that $A_{i} \hookrightarrow A_{j}$, by 2.2 . Since there are only finitely many $i$ we must have $A_{k} \hookrightarrow A_{j} \hookrightarrow A_{k}$ for some indices $k$ and $j$ with $k \neq j$. It follows from [3,6.2(d)] that $A_{k} \dot{\sim} A_{j}$ which is a contradiction. Thus, all the $A_{i}$ are pairwise quasi-isomorphic and every nonzero $\varphi \in \operatorname{Hom}\left(A_{i}, A_{j}\right)$ is monic. This implies that every nonzero endomorphism of $A_{i}$ is a 
monomorphism and $Q E\left(A_{i}\right)$ is a division algebra $[3,6.1]$ as claimed. The equivalence of (2) and (3) is due to Reid [19]. Assume the validity of (3), let $G \doteq H \oplus K$ and let $\varepsilon: H \rightarrow K$ be a nonzero homomorhism. Note that $G \dot{\simeq} A^{n}$ with $A$ strongly indecomposable, and Jónsson's theorem implies $H \dot{\sim} A^{p}$ and $K \dot{\sim} A^{q}$ for some positive integers $p$ and $q[14,92.5]$. By Reid's result, both $H$ and $K$ have simple quasiendomorphism rings. Let $L=\varepsilon H$. Then $L=S_{H}(L) \doteq S_{A}(L) \doteq S_{K}(L)$. Applying 3.1(2) with $W=K$ we conclude that $L$ is a quasi-summand of $K$. Hence, $L \dot{\sim} A^{m}$ for some positive integer $m$. It follows that $S_{L}(H) \doteq S_{A}(H) \doteq H$ and that $Q E(L)$ is a simple ring, too. Aplying 3.1(3) with $W=L$ completes the proof.

We now characterise the almost completely decomposable groups with q-SIP. The first step toward this goal is the following lemma. The type of a rank-one group $X$ is denoted by $t(X)$.

Lemma 3.3. Let $A, B$ and $C$ be rank-one groups such that $t(A) \leqslant t(C)$ and $t(B) \leqslant t(C)$, and let $G \doteq A \oplus B \oplus C$. If $G$ has $q-S I P$ then $t(A)$ and $t(B)$ are comparable.

Proof: Pick nonzero homomorphisms $\alpha: A \rightarrow C$ and $\beta: B \rightarrow C$ and define $\varepsilon: A \oplus B \rightarrow C$ by $\varepsilon(a, b)=\alpha(a)-\beta(b)$. Let $X=\alpha(A) \cap \beta(B)$. Then $X$ is a rankone group and $t(X)$ is a lower bound of $t(A)$ and $t(B)$. Furthermore, kernel $(\varepsilon)=$ $\left\{\left(\alpha^{-1}(x), \beta^{-1}(x)\right) \mid x \in X\right\} \simeq X$, and kernel $(\varepsilon)$ is a quasi-summand of $A \oplus B$, by 2.2 . Thus, $X \simeq A$ or $X \simeq B$ as desired.

The completely decomposable groups $G$ that are flat as modules over their endomorphism rings have been characterised by Arnold [4] and Richman and Walker [20]: if $T$ denotes the set of types of the rank-one summands of $G$ then $G$ is a flat $E(G)$ module if and only if $T$ has the property that any two of its elements that have an upper bound in $T$ must also have a lower bound in $T$. Combining this with our results we obtain

THEOREM 3.4. A completely decomposable torsion-free group $G$ has $q$-SIP if and only if (i) its endomorphism ring $E=E(G)$ has q-SIP as a right regular module and (ii) $G$ is flat as a left $E$-module.

Proof: Sufficiency of (i) and (ii) follows from 2.8. For the converse, let $G=$ $\bigoplus_{i \in I} X_{i}$ with each $X_{i}$ of rank one and type $t_{i}$, let $T=\left\{t_{i}\right\}_{i \in I}$, and suppose $G$ has q-SIP. By 2.7 , so does $E_{E}$ and, for any three pairwise distinct $i, j, k$ in $I$, so does $X_{i} \oplus X_{j} \oplus X_{k}$. By 3.3 and [20, Theorem 4], $G$ is $E$-flat.

We have a similar result for almost completely decomposable groups:

THEOREM 3.5. An almost completely decomposable torsion-free group $G$ has $q$-SIP if and only if (i) its endomorphism ring $E=E(G)$ has $q-S I P$ as a right regular module and (ii) $G$ is quasi-equal to a group $H$ which is flat as a left $E(H)$-module. 
Proof: Combine 3.4 and 2.3 .

For almost completely decomposable groups of finite rank the quasi-summand intersection property can be characterised by the set of types of the quasi-summands:

THEOREM 3.6. Let $\left\{X_{i}\right\}_{i=1}^{n}$ be a family of torsion-free rank-one groups $X_{i}$ of type $t_{i}$, let $T=\left\{t_{i} \mid i=1, \ldots, n\right\}$, and let $G \doteq X_{1} \oplus \cdots \oplus X_{n}$ with endomorphism ring $E=E(G)$. Then $G$ has q-SIP if and only if any two types in $T$ which have an upper bound in $T$ are comparable.

Proof: By 3.3, the stated condition is necessary for $T$. Conversely, assume $T$ is as stated but $G$ fails to have q-SIP. Choose a counterexample, $G$, of minimal rank. Following Bowman and Rangaswamy [8], define an equivalence relation on $T$ by letting $\sigma \sim \tau$ if there exist $\tau_{1}, \ldots, \tau_{m} \in T$ such that $\sigma=\tau_{1}, \tau=\tau_{m}$, and $\tau_{i}$ and $\tau_{i+1}$ are comparable for $i=1, \ldots, m-1$. If $G_{[t]}=\bigoplus_{t_{i} \in[t]} X_{i}$ then each $G_{[t]}$ is fully invariant in $G$ and $G$ is quasi-equal to the direct sum of the $G_{[t]}$. By minimality, $G \doteq G_{[t]}$ and any two types in $T$ are equivalent. Let $\sigma \in T$ be a minimal element. As in [15, p.147], the hypothesis on $T$ implies that $\sigma \leqslant t$ for all $t \in T$. Let $H=\bigoplus_{t_{i}=\sigma} X_{i}$ and $F=\bigoplus_{i_{j}>\sigma} X_{j}$. Then $G \doteq H \oplus F$ and $F$ is fully invariant in $G$. Since $G$ is a counterexample, $G$ has a quasi-decomposition $G \doteq A \oplus B$ for which there is a homomorphism $\varepsilon: A \rightarrow B$ such that $K=\operatorname{kernel}(\varepsilon)$ is no quasi-summand of $A$; among all such decompositions choose one in which $A$ has minimal rank, p. Note that $F \doteq(F \cap A) \oplus(F \cap B)[3,2.1]$. By Jónssen's result, $A \doteq A_{1} \oplus \cdots \oplus A_{p}$ with each $A_{k} \simeq X_{i}$ for some $i$; in addition, the minimality of the rank of $A$ implies that each restriction map $\varepsilon \mid A_{j}$ is monic, $j=1, \ldots, p$. If $A \leqslant F$ then $F \doteq A \oplus(F \cap B)$ and $\varepsilon(A) \leqslant F \cap B$ so that $K$ is a quasi-summand of $A$ since $F$ has a rank smaller than the rank of $G$. Consequently, we may assume that $t\left(A_{1}\right)=\sigma$. Put $W=\bigoplus_{i=2}^{p} A_{i}$. Since $W \oplus B$ has a rank that is smaller than the rank of $G, W \oplus B$ has q-SIP so that $K \cap W$ is a quasi-summand of $W$. Again, the minimality of the rank of $A$ implies that $\varepsilon \mid W$ is monic. Hence $\varepsilon(A)=\varepsilon\left(A_{1}\right)+\varepsilon(W)$ with $\varepsilon\left(A_{1}\right) \simeq A_{1}$ and $\varepsilon(W) \simeq W$. Suppose $\varepsilon\left(A_{1}\right) \cap \varepsilon(W)=0$. Then $\varepsilon$ is monic which is impossible. Consequently, $\varepsilon\left(A_{1}\right) \cap \varepsilon(W) \neq 0$ and $\varepsilon\left(A_{1}\right) \cap \varepsilon(W)$ has rank one. Since $t(x) \geqslant \sigma=t\left(A_{1}\right)$ for all $x \in G$, it follows that $t\left(A_{1}\right)=t\left(\varepsilon\left(A_{1}\right)\right)=t\left(\varepsilon\left(A_{1}\right) \cap \varepsilon(W)\right)$. Hence $m \varepsilon\left(A_{1}\right) \leqslant \varepsilon(W)$ for some positive integer $m[3,6.1]$ which implies $\varepsilon(A) \doteq \varepsilon(W)$. But then $A \doteq W+K=W \oplus K$ and the proof is completed.

We note that 3.6 does not hold for groups of infinite rank: if $F$ is a free group of infinite rank and $A$ is any torsion-free group of rank one which is not isomorphic to $Z$ then $G=F \oplus A$ does not have q-SIP even though the set $T=\{t(Z), t(A)\}$ is linearly ordered. 


\section{EXAMPLES}

By 2.7, the quasi-summand intersection property of the module implies that of its endomorphism ring. The converse of this is not true. In fact, we can make a stronger statement (see 2.4):

EXAMPLE 4.1. There exists a torsion-free abelian group $G$ of rank three without $q$-SIP whose quasi-endomorphism ring $Q E(G)$ is right principal projective.

ProOF: Let $R$ be the set of all rationals with denominator a power of 2 , let $S$ be the set of all rationals with denominator a power of three, and let $T=R+S$ as a subset of $Q$. Then $R, S$ and $T$ are additive groups as well as subrings of $Q$. Let $G=R \oplus S \oplus T$. It follows from 3.3 that $G$ does not have q-SIP. Furthermore

$$
Q E(G) \simeq Q\left(\begin{array}{ccc}
R & 0 & 0 \\
0 & S & 0 \\
T & T & T
\end{array}\right)=\left(\begin{array}{lll}
Q & 0 & 0 \\
0 & Q & 0 \\
Q & Q & Q
\end{array}\right)
$$

which is easily verified to be right principal projective as desired.

According to 2.7, a module with q-SIP satisfies some mild flatness conditions over its endomorphism ring. These conditions by themselves do not imply q-SIP, in fact, even $E$-flatness does not imply q-SIP:

EXAMPLE 4.2. There exists a torsion-free abelian group $A$ of rank four which is flat as a module over its endomorphism ring but does not have q-SIP.

Proof: Let $A=Z \oplus G$ where $G$ is the group of 4.1. Since $G$ does not have q-SIP, neither does $A$. By [20, Theorem 4], $A$ is flat over its endomorphism ring.

Conversely, modules with q-SIP need not be flat. In order to provide an example we will use recent results on the representation of rings as endomorphism rings. Since Corner's celebrated theorem that every countable reduced torsion-free ring is the endomorphism ring of a countable reduced torsion-free group [11], significant progress has been made on this subject. Recently, a more delicate question has been considered: given a ring $R$ and some module-theoretic property $\mathcal{N}$, is there a group $G$ such that $E(G) \simeq R$ and, in addition, the left $E(G)$-module $G$ has property $\mathcal{N}$ ? Faticoni and Goeters [13] and Albrecht [2] considered this problem for $\mathcal{N}$ being the property "flat". Every finite-rank torsion-free abelian group with right semihereditary endomorphism ring must be $E$-flat [6], [16]; but if $R$ is a reduced torsion-free ring of finite rank which is not semihereditary, $R$ can be realised as the endomorphism ring of a finite-rank torsion-free group which is not flat over its endomorphism ring [13, 3.11]. In contrast, both Corner's construction and the Dugas-Göbel construction [12] lead to $E$-flat groups $[13,2]$. 
EXAMPLE 4.3. There exists a torsion-free abelian group $A$ which has $q$-SIP but is not flat as a left $E(A)$-module.

Proof: Consider the subring $R=Z \oplus 2 i Z$ of the field of complex numbers, $i=\sqrt{-1}$. It is well known that $R$ is not semihereditary. By [13] there exists an abelian group $A$ with $E(A) \simeq R$ which is not flat as a left $E(A)$-module. Since $Q R=Q(i)$ is a field, $A$ is strongly indecomposable.

Examples of finite-rank torsion-free groups that fail to have SIP but do have q-SIP exist in abundance (see for example [15, p.144]). Modules with SIP that do not have q-SIP are less common. We have

EXAMPLE 4.4. There exists a torsion-free abelian group $A$ of finite rank which has SIP but not $q-S I P$.

Proof: Consider the matrix ring $\mathcal{R}=\left\{\left(\begin{array}{ll}x & 0 \\ y & x\end{array}\right) \mid x, y \in Z\right\}$. Its additive group is free of rank two, and $Q \mathcal{R}$ is a commutative local ring with unique maximal ideal $\mathcal{J}=\left(\begin{array}{ll}0 & 0 \\ Q & 0\end{array}\right)$. Thus, the regular module $Q \mathcal{R}$ is indecomposable. One verifies that $\mathcal{J}$ equals the annihilator ideal of the matrix $T=\left(\begin{array}{ll}0 & 0 \\ 1 & 0\end{array}\right) \in Q \mathcal{R}$. Let $\mathcal{M}=M_{2}(\mathcal{R})$ be the ring of $2 \times 2$-matrices over $\mathcal{R}$, and consider its subring $\mathcal{S}=Z \cdot 1_{\mathcal{M}}+2 \mathcal{M}$. Then $\mathcal{S} \doteq \mathcal{M}$, and their additive groups are free of finite rank. There exists a torsion-free abelian group $A$ of finite rank such that $E(A) \simeq \mathcal{S}$ and $A$ is flat over its endomorphism ring $[13,2]$. The quasi-endomorphism ring of $A$ is isomorphic to $Q \mathcal{M}=M_{2}(Q \mathcal{R})$. We claim that $A$ has SIP but not q-SIP. For the latter, it suffices to show that the regular module $Q \mathcal{M}$ fails to have SIP (2.7). Let $\pi=\left(\begin{array}{ll}1 & 0 \\ 0 & 0\end{array}\right) \in Q \mathcal{M}$ and let $\Phi$ : $\pi Q \mathcal{M} \rightarrow(1-\pi) Q \mathcal{M}$ be the left multiplication by the matrix $\left(\begin{array}{ll}0 & 0 \\ T & 0\end{array}\right) \in Q \mathcal{M}$. One verifies that the kernel of $\Phi$ equals $\mathcal{K}=\left(\begin{array}{cc}\mathcal{J} & \mathcal{J} \\ 0 & 0\end{array}\right)$ which is not a direct summand of the $Q \mathcal{M}$-module $\pi Q \mathcal{M}=\left(\begin{array}{cc}Q \mathcal{R} & Q \mathcal{R} \\ 0 & 0\end{array}\right) . \mathrm{By}[15,1.4], Q \mathcal{M}$ fails to have SIP as a right regular module. The proof is completed once we show $\mathcal{S}$ has no nontrivial idempotents. Assume $\rho=\rho^{2} \in \mathcal{S}$. Then $\rho=n \cdot 1+2 \alpha$ for some $\alpha \in \mathcal{M}$ and an integer $n \in\{0,1\}$. Computation yields $0=n(1-n) \cdot 1=4 \alpha^{2}+(4 n-2) \alpha$. It follows that, for both values of $n, \alpha= \pm 2 \alpha^{2}$ so that $\alpha$ has infinite 2-height in $\mathcal{M}$. By the freeness of $\mathcal{M}$ we must have $\alpha=0$ which shows $\rho$ is trivial. This completes the proof.

We now show that the quasi-summand intersection property of a module cannot be characterised by its endomorphism (or its quasi-endomorphism) ring alone:

EXAMPLE 4.5. There exist two $Z$-modules with isomorphic endomorphism 
rings one of which has q-SIP while the other one does not.

Proof: Let $G$ be the group in Example 4.1 and let $E$ be its endomorphism ring. Then $G$ does not have the quasi-summand intersection property while $E_{E}$ does. Clearly, $E$ is a countable reduced torsion-free ring. By [13], Corner's construction in [11] realises $E$ as the endomorphism ring of a torsion-free group $H$ such that $H$ is flat as a left $E(H)$-module. Since $E(H) \simeq E$, it follows from 2.8 that $H$ has q-SIP.

\section{REFERENCES}

[1] U. Albrecht, 'Baer's Lemma and Fuchs' Problem 84a', Trans. Amer. Math. Soc. 293 (1986), 565-582.

[2] U. Albrecht, 'Endomorphism rings of faithfully flat abelian groups', Results Math. 17 (1990), 179-201.

[3] D. M. Arnold, Finite rank torsion free abelian groups and rings: Lecture Notes in Mathematics 931 (Springer-Verlag, Berlin, Heidelberg, New York, 1982).

[4] D. M. Arnold, 'Abelian groups as flat modules over their endomorphism rings', unpublished lecture notes.

[5] D. M. Arnold and J. Hausen, 'A characterization of modules with the summand intersection property', Comm. Algebra (to appear).

[6] D.M. Arnold and E. L. Lady, 'Endomorphism rings and direct sums of torsion free abelian groups', Trans. Amer. Math. Soc. 211 (1975), 225-237.

[7] R. A. Beaumont and R. S. Pierce, 'Torsion-free rings', Illinois J. Math. 5 (1961), 61-98.

[8] H. Bowman and K. M. Rangaswamy, 'Torsion-free separable abelian groups quasiprojective over their endomorphism rings', Houston J. Math. 11 (1985), 447-453.

[9] H. Cartan and S. Eilenberg, Homological Algebra (Princeton University Press, Princeton, New Jersey, 1956).

[10] A. W. Chatters and C. R. Hajarnavis, Rings with Chain Conditions (Pitman, London, 1980).

[11] A. L. S. Corner, 'Every countable reduced torsion-free ring is an endomorphism ring', Proc. London Math. Soc. 13 (1963), 687-710.

[12] M. Dugas and R. Göbel, 'Every cotorsion-free ring is an endomorphism ring', Proc. London Math. Soc. 45 (1982), 319-336.

[13] T. G. Faticoni and H. P. Goeters, 'Examples of torsion-free groups flat as modules over their endomorphism rings', Comm. Algebra 19 (1991), 1-27.

[14] L. Fuchs, Infinite Abelian Groups, II (Academic Press, New York, 1973).

[15] J. Hausen, 'Modules with the summand intersection property', Comm. Algebra 17 (1989), 135-148.

[16] M. Huber and R. B. Warfield, Jr., 'Homomorphisms between cartesian powers of an abelian group', in Abelian Group Theory: Lecture Notes in Mathematics 874, pp. 202-227 (Springer-Verlag, New York, 1981).

[17] B. Jónssen, 'On direct decompositions of torsion free abelian groups', Math. Scand. 7 
(1959), 361-371.

[18] I. Kaplansky, Infinite Abelian Groups (revised edition)(University of Michigan Press, Ann Arbor, 1969).

[19] J. D. Reid, 'On quasi-decompositions of torsion free abelian groups', Proc. Amer. Math. Soc. 13 (1962), 550-554.

[20] F. Richman and E. A. Walker, 'Cyclic Ext', Rocky Mountain J. Math. 11 (1981), 611-615.

[21] F. Ulmer, 'Localizations of endomorphism rings and fixpoints', J. Algebra 43 (1976), 529-551.

[22] G. V. Wilson, 'Modules with the summand intersection property', Comm. Algebra 14 (1986), 21-38.

Department of Mathematics

Auburn University

Auburn AL 36849-5307

United States of America
Department of Mathematics

University of Houston

Houston TX 77204-3476

United States of America 